\title{
RISCOS OCUPACIONAIS EM UM SETOR DE HEMODIÁLISE NA PERSPECTIVA DOS TRABALHADORES DA EQUIPE DE ENFERMAGEM
}

\author{
Occupational risks in a unit of hemodialysis in the \\ perspective of the workers of the nursing team
}

\author{
Riesgos ocupacionales en una unidad de hemodialisis en el \\ perspectiva de los trabajadores del equipo de enfermería
}

Michele Karla Damacena da Silva ${ }^{1}$

Regina Célia Gollner Zeitoune²

\section{RESUMO}

Estudo descritivo exploratório, de abordagem qualitativa, teve como objetivos descrever os riscos ocupacionais no contexto dos trabalhadores da equipe de enfermagem em uma unidade de hemodiálise, analisar o conhecimento do trabalhador da equipe de enfermagem acerca das medidas de proteção e segurança em uma unidade de hemodiálise e discutir o conhecimento do trabalhador da equipe de enfermagem sobre os riscos ocupacionais e as implicações para a saúde do trabalhador. 0 cenário foi um hospital universitário do município do Rio de Janeiro. Os sujeitos foram 26 trabalhadores da equipe de enfermagem do setor de hemodiálise. Foi utilizada entrevista semiestruturada. 0 projeto foi aprovado por Comitê de Ética. Resultados: os trabalhadores detêm o conhecimento sobre os riscos ocupacionais e sobre as medidas de proteção e segurança, apesar de nem sempre aplicálas na sua prática profissional. Foram citados como as principais implicações à saúde os problemas respiratórios, de coluna e as doenças contagiosas.

Palavras-chave: Saúde do Trabalhador. Riscos Ocupacionais. Enfermagem. Diálise Renal.

\begin{abstract}
Exploratory descriptive study, of qualitative approach, which had as objective to describe the occupational risks in the context of the workers of the nursing staff in a unit of hemodialysis, to analyze the knowledge of the worker of the nursing staff concerning the measures of protection and security in a unit of hemodialysis and to argue the knowledge of the worker of the nursing staff about the occupational risks and the implications for the health of the worker. The scenery was a University Hospital of the city of Rio de Janeiro (Brazil). The citizens had been 26 workers of the nursing staff of the hemodialysis' sector. Halfstructuralized interview was used. The project was approved by the Ethic Committee. Results: the workers withhold the knowledge about occupational risks and the measures of protection and security, although not always apply them in their professional practice. The respiratory problems were cited as the main implications to the health, of spine and the contagious diseases.
\end{abstract}

Keywords: Occupational Health. Occupational Risks. Nursing. Renal Dialysis.

\section{Resumen}

Estudio descriptivo exploratorio, de abordaje cualitativo, que tiene como objetivo describir los riesgos ocupacionales en el contexto de los trabajadores del equipo de enfermería en una unidad de hemodialisis, analizar el conocimiento del trabajador del equipo de enfermería a respecto de las medidas de protección y seguridad en una unidad de hemodialisis y discutir el conocimiento del trabajador del equipo de enfermería sobre los riesgos ocupacionales y las implicaciones para la salud del trabajador. El escenario fue un Hospital Universitario de la ciudad de Rio de Janeiro (Brasil). Los sujetos fueron 26 trabajadores del equipo de enfermería del sector de hemodialisis. Fue utilizada la entrevista semi-estructurada. El proyecto fue aprobado por el Comité de Ética. Resultados: los trabajadores retienen el conocimiento sobre los riesgos ocupacionales y sobre las medidas de protección y seguridad, aunque ni siempre aplicarlas en su práctica profesional. Los problemas respiratorios fueron citados como las implicaciones principales a la salud, de columna y las enfermedades contagiosas.
Palabras clave: Salud Laboral. Riesgos Laborales. Enfermería. Diálises Renal.

'Mestranda do Curso de Pós-Graduação da Escola de Enfermagem Anna Nery (EEAN) da Universidade Federal do Rio de Janeiro (UFRJ) Especialista em Nefrologia pela Universidade do Estado do Rio de Janeiro. Especialista em Enfermagem do Trabalho pela EEAN/UFRJ. Enfermeira líder do Hospital Universitário Clementino Fraga Filho/UFRJ. Brasil. E-mail: micheledamacena@yahoo.com.br, ${ }^{2}$ Professora Adjunta do Departamento de Enfermagem de Saúde Pública (DESP) da EEAN/UFRJ. Doutora em Enfermagem. Pesquisadora do Núcleo de Pesquisa Enfermagem e Saúde do Trabalhador/DESP/EEAN/UFRJ. Brasil. E-mail: regina.zeitoune@gmail.com 


\section{INTRODUÇÃO}

0 presente estudo aborda os riscos ocupacionais existentes em uma unidade de hemodiálise (HD). Na realidade estudada, considerando a rotatividade na equipe de auxiliares e técnicos de enfermagem do referido setor, cabe chamar atenção à necessidade de acompanhamento e treinamento específicos destes profissionais, devido à complexidade do setor e peculiaridade dos clientes renais crônicos, que demandam procedimentos específicos e uso de medidas de proteção e segurança à saúde dos trabalhadores.

A partir desta vivência, as questões sobre a exposição aos riscos ocupacionais tornaram-se uma inquietação a ser avaliada. Neste contexto, surgiu o questionamento com relação à percepção dos trabalhadores da equipe de enfermagem sobre os riscos ocupacionais aos quais estão expostos regularmente em uma unidade de HD.

Os objetivos traçados buscaram descrever os riscos ocupacionais no contexto dos trabalhadores da equipe de enfermagem em uma unidade de HD; analisar o conhecimento do trabalhador da equipe de enfermagem acerca das medidas de proteção e segurança em uma unidade de HD; e discutir o conhecimento do trabalhador da equipe de enfermagem sobre os riscos ocupacionais e as implicações para a saúde do trabalhador.

\section{REFERENCIAL TEÓRICO}

Por definição, a saúde do trabalhador é entendida como o processo saúde e doença dos grupos humanos, em sua relação com o trabalho, representando um esforço de compreensão deste processo - como e por que ocorre - e do desenvolvimento de alternativas de intervenção que levem à transformação em direção à apropriação pelos trabalhadores da dimensão humana do trabalho, numa perspectiva teleológica'.

Historicamente, os trabalhadores da área da saúde não eram considerados como categoria profissional de alto risco para acidentes de trabalho. A preocupação com os riscos biológicos surgiu somente a partir da epidemia de HIV/AIDS nos anos 80 , quando foram estabelecidas normas para as questões de segurança no ambiente de trabalho.

Os agravos à saúde relacionados ao trabalho são classificados em dois grupos: no primeiro incluem-se aqueles que traduzem uma ruptura abrupta do equilíbrio entre as condições e o ambiente de trabalho e a saúde do trabalhador, como os acidentes de trabalho e as intoxicações agudas de origem profissional. 0 segundo grupo inclui agravos de caráter crônico: a doença profissional típica, definida como aquela inerente ou peculiar a determinado ramo de atividade ${ }^{2}$.

Um outro grupo é constituído pelas doenças relacionadas com o trabalho, definidas pela Organização Mundial de Saúde (OMS) como agravos outros que, em adição a doenças profissionais legalmente desconhecidas, ocorrem em trabalhadores quando o ambiente ou as condições contribuem significativamente para a ocorrência de doenças, porém em graus variados de magnitude 3 .

De acordo com a Norma Regulamentadora $n^{\circ} 9$ (NR-9) do Ministério do Trabalho e Emprego (MTE), consideram-se riscos ambientais os agentes físicos, químicos e biológicos existentes nos ambientes de trabalho, que, em função de sua natureza, concentração ou intensidade e tempo de exposição, são capazes de causar danos à saúde do trabalhador ${ }^{4}$.

Há ainda os riscos ergonômicos, gerados principalmente pela postura irregular dos profissionais de enfermagem em situações como movimentações de pacientes, flexões da coluna frequentes, entre outros ${ }^{5}$.

Já os riscos de acidentes são aqueles inerentes a cada ambiente de trabalho, como, por exemplo, risco de queda decorrente de pisos ou iluminação inadequados.

0 ambiente hospitalar apresenta uma diversidade de profissionais de saúde e trabalhadores treinados para realizar variadas atividades necessárias à manutenção da estrutura da instituiçã $0^{6}$. Neste contexto, a equipe de enfermagem constitui a maior representatividade de pessoal dentro do hospital, e sua atividade primordial caracteriza-se na promoção, prevenção e recuperação da saúde de um número elevado de pessoas.

Para controle e diminuição da ocorrência de acidentes e doenças ocupacionais, torna-se imprescindível a realização de educação em saúde para todos os trabalhadores envolvidos com o setor. A educação, no ambiente de trabalho, serve de suporte para que os trabalhadores possam desempenhar suas funções com mais segurança e qualidade, constituindo-se uma exigência de todos os serviços de assistência à saúde ${ }^{6}$.

0 processo de trabalho em HD envolve um contato íntimo com fluidos orgânicos, expondo os trabalhadores a diversos patógenos. Diante disso, a adoção de medidas de biossegurança é, de fato, muito importante para que haja uma prevenção efetiva de acidentes e doenças ocupacionais advindas dos riscos biológicos. Para isso, o uso de equipamentos de proteção individual (EPI), como máscara, gorro, óculos, capote, botas, entre outros, torna-se obrigatório a todos os trabalhadores.

0 comprometimento da adequada realização destas medidas proporciona maior proteção contra agravos à saúde ocupacional.

\section{METODOLOGIA}

A abordagem do estudo foi qualitativa, descritiva, do tipo exploratória. Foi realizado em um hospital universitário do município do Rio de Janeiro, na unidade de HD, localizada no $7^{\circ}$ andar, que integra o setor de Nefrologia juntamente com a unidade de Transplante Renal e Diálise Peritoneal.

0 projeto de pesquisa foi previamente enviado e analisado e devidamente aprovado pelo Comitê de Ética e Pesquisa do Hospital Universitário da Universidade Federal do Rio de Janeiro, sob o número 106/07. Além disso, todos os sujeitos que se disponibilizaram a participar do estudo, leram e 
assinaram o Termo de Consentimento Livre e Esclarecido, de acordo com a Resolução 196/96.

Foi entrevistado um total de 26 sujeitos, caracterizados por 5 enfermeiros, 16 técnicos de enfermagem e 5 auxiliares de enfermagem, todos integrantes da equipe de enfermagem do setor de HD; 46,1\% deles tinham entre 41 e 50 anos de idade; $88,5 \%$ eram do sexo feminino; $61,5 \%$ eram pertencentes à categoria de técnico de enfermagem; 84,6\% relataram ter até cinco anos de serviço tanto no hospital como no setor de HD do hospital $(85,6 \%)$ e também como tempo de experiência total em HD $(69,2 \%)$.

Quanto aos treinamentos sobre riscos ocupacionais, 53,9\% relataram terem recebido alguma orientação fora do hospital em estudo e 42,3\% nunca tiveram. Já em relação a treinamentos sobre medidas de proteção e segurança, 73,1\% relataram terem sido orientados fora do hospital e $65,4 \%$, no hospital em estudo.

0 instrumento de coleta de dados foi a entrevista semiestruturada com três perguntas. As entrevistas foram realizadas no próprio setor de $\mathrm{HD}$ e gravadas digitalmente, e os dados foram transcritos na íntegra. Foi mantido o anonimato dos sujeitos, utilizando apenas as iniciais de cada categoria profissional, seguidas do número da respectiva entrevista.

A análise dos dados foi realizada a partir do surgimento da saturação dos dados coletados. Utilizou-se a técnica de análise temática, na qual foi possível a formulação de três categorias, descritas adiante.

\section{ANÁLISE DOS RESULTADOS}

A apresentação, a análise e a interpretação dos resultados desenvolveram-se de acordo com os objetivos do estudo e foram estruturadas em três categorias, conforme descrição a seguir.

\section{Riscos ocupacionais e o contexto da hemodiálise}

Nesta categoria, os sujeitos do estudo apontaram os riscos ocupacionais que eles percebiam existir no seu contexto de trabalho. É sabido que a complexidade de uma unidade de hemodiálise expõe os trabalhadores de enfermagem a múltiplos riscos ocupacionais, conforme descritos pelo MTE ${ }^{4}$.

Dentre os riscos ocupacionais percebidos pelos profissionais de enfermagem, está o biológico, que, considerando ser 0 hospital o ambiente de trabalho, se tornou mais perceptível, como se observa nas falas:

tem o risco biológico, risco de se contaminar com material perfurocortante[...] (E1)

A enfermagem está exposta a várias patologias, como AIDS, hepatites $B$ e C, BK; há também o risco de infecção por MARSA[...] Também lidamos com sangue, secreções, [...] e o risco sempre presente com os perfurocortantes. (T10)
A exposição aos riscos biológicos é preocupante, uma vez que são causadores de muitos problemas de saúde dos trabalhadores, pois, ao executarem atividades que envolvem 0 cuidado direto e indireto aos pacientes, estão frequentemente expostos às infecções transmitidas por micro-organismos presentes no sangue ou outros fluidos orgânicos.

A NR-9, do MTE, considera como agentes biológicos as bactérias, fungos, bacilos, parasitas, protozoários, vírus, entre outros 4 .

Dentre os acidentes que podem ocorrer no ambiente hospitalar, os que envolvem os materiais perfurocortantes, em especial as agulhas, têm sido reconhecidos como um dos principais problemas de exposição para os trabalhadores na aquisição de infecções, expondo-os também aos riscos de acidentes $^{7,8}$

Os acidentes ocasionados por picada de agulhas são responsáveis por $80 \%$ a $90 \%$ das transmissões de doenças infecciosas entre trabalhadores de saúde. 0 risco de transmissão de infecção através de uma agulha contaminada é de $1 \mathrm{em} 3$ para hepatite $B, 1$ em 30 para hepatite $C$ e 1 em 300 para HIV $^{8}$.

Os trabalhadores de enfermagem do setor estudado lidam continuamente com uma clientela de características muito particulares, muitas vezes portadores do vírus HIV, da hepatite $\mathrm{Be} C$ e muitos outros com precauções respiratórias por $\mathrm{BK}$ e de contato. Inclusive há pacientes com precaução de contato por MARSA resistente a Mupirocin (MARSA Mupirocin-R), que estão em precaução de contato por tempo indeterminado.

A vacinação para hepatite $B$ tem contribuído bastante para proteger os trabalhadores e os próprios pacientes. Quanto ao HIV e à hepatite $C$, não existe imunoprofilaxia pré-exposição $e$ também nenhuma medida espećíica para a redução do risco de transmissão pós-exposição ao vírus ${ }^{8}$.

0 risco químico também esteve evidente em algumas entrevistas, pois estes trabalhadores permaneceram em contato próximo, diariamente, com uma série de substâncias químicas, como o hipoclorito de sódio a $2 \%$ e o proxitane, as quais são utilizadas para desinfecção das máquinas de HD e esterilização dos dialisadores dos pacientes, como se observa:

[...] também o cheiro da substância química[...]o proxitane é muito forte; na hora de preparar a solução ou na hora de colocar a solução no sistema[...] nós corremos o risco de inalar este cheiro e ser prejudicial aos nossos pulmões. (T13)

[...] tem o reuso[...] aquele cheiro do proxitane é muito forte, daí irrita as vias aéreas. (T12)

A legislação vigente (NR-9, MTE) refere que devemos considerar como agentes químicos as substâncias, compostos ou produtos que possam penetrar no organismo pela via 
respiratória, nas formas de poeira, fumo, névoas, neblina, gases ou vapores, ou que, pela natureza da atividade de exposição, possam ter contato ou ser absorvidos pelo organismo através da pele ou por ingestão $0^{4}$.

Como foi referido, o hospital apresenta uma variedade de riscos, entre os quais os químicos, que parecem estar relacionados mais intensamente com as drogas antineoplásicas, os agentes anestésicos, os agentes esterilizantes e os irritantes de pele. Esses agentes químicos são capazes de provocar nos trabalhadores intoxicações agudas e estados crônicos e prépatológicos de várias doenças ocupacionais9.

Além disso, a saúde humana dos trabalhadores expostos a substâncias químicas está sujeita a uma multiplicidade de possíveis efeitos, tais como a teratogênese, a carcinogênese, a mutagênese e danos a órgãos-alvo específicos ${ }^{10}$.

Pelo fato de os agentes químicos não serem tão visíveis, os próprios profissionais não dão a necessária atenção aos cuidados de segurança ao manipulá-los, como podemos observar na seguinte citação:

[...] no começo eu sentia o meu nariz arder, porque depois acostuma[...], mas o começo[...]. No começo eu sentia mais, agora meio que me acostumei, mas [...] ele continua aqui presente, a gente é que não tá percebendo. (T26)

Portanto, é necessário que o hospital tenha um serviço de saúde do trabalhador efetivo não só na fiscalização da utilização dos produtos, armazenamento e descarte, mas também realizando treinamentos aos trabalhadores que lidam constantemente com estes materiais e programas de controle biológico da exposição aos agentes químicos.

Um outro risco ocupacional apontado foi o risco ergonômico, o qual pode ser observado nas falas a seguir:

[...] tem também o fator da postura; hoje a gente vê que muitas de nossas colegas estão sofrendo as consequências por causa da má postura [...] (A24)

[...]é pegar peso, esses galões, as bolsas de diálise [...] é[...] abaixar e levantar repetitivas vezes $[\ldots](T 7)$

Este risco está presente possivelmente como consequência das adaptações não ergonômicas do próprio setor e, consequentemente, da necessidade que o trabalhador tem de se adaptar à realidade de trabalho, que o leva a adotar posturas inadequadas e viciosas, o que provavelmente produzirá efeitos maléficos à sua saúde.

No dia-a-dia hospitalar, os esforços de auxílio ao paciente com dificuldade de locomoção e até para se levantar do leito, a passagem de macas ao leito e vice-versa, os períodos de jornadas, os horários noturnos, a iluminação artificial predominante, a deambulação com posições viciosas, os movimentos repetitivos, entre outros, justificam os problemas de colunas, entre outros, relatados pelos trabalhadores ${ }^{11}$.

0 risco de acidentes foi também mencionado, em uma menor dimensão, pelos entrevistados, como podemos observar a seguir:

É[...] risco de se acidentar dentro do ambiente de trabalho, pela correria com que fazem as coisas...eu acho que a atenção fica muito diminuída pela falta de tempo e pela pressa em desenvolver a atividade, então isso reduz a atenção, aumentando o risco de cair, de escorregar.se machucar e ter acidentes feios. (E9)

No setor em questão, este risco está presente de forma regular, visto que os profissionais de enfermagem trabalham 24 horas por dia, e o setor necessita ser lavado e higienizado com os trabalhadores no setor. Além disso, o próprio maquinário utilizado na HD propicia vazamentos constantes quando apresentam algum dano, até mesmo pelo seu uso contínuo.

\section{Conhecendo as medidas de proteção e segurança diante dos riscos ocupacionais}

As medidas de proteção e segurança são formas de prevenção a serem utilizadas na assistência a todos os pacientes na manipulação de sangue, secreções e excreções e no contato com mucosas e pele não íntegra.

Tais medidas incluem a utilização de EPIs, com a finalidade de reduzir a exposição ao sangue ou fluidos corpóreos, e os cuidados específicos recomendados para manipulação e descarte de materiais contaminados por material orgânico ${ }^{12}$.

Nesta categoria, observou-se que os trabalhadores têm 0 conhecimento sobre as medidas de prevenção e segurança, principalmente quanto aos equipamentos de proteção individual, conforme as falas a seguir:

Ah[...] você tem que trabalhar sempre de óculos, nunca tirar, principalmente quando vai manipular cateter, porque aquilo ali espirra[...] principalmente na HD[...] colocar o avental de plástico[...] máscara, luvas sempre[...] eu acho que todo material aqui na HD tem que ser usado, não dá pra abrir mão de nenhum. (T5)

Uso de máscara, de avental, de luva, máscara de carvão ativado para os gases do proxitane[...] e os cuidados com os perfurocortantes[...](A23)

Em conformidade com o que foi mencionado, uma das formas de evitar acidentes ocupacionais é o uso de EPI, que 
constitui uma barreira protetora para o trabalho, pois reduz efetivamente, mas não elimina, o risco de exposição ocupacional.

No caso do setor em estudo, os EPIs indicados são gorro, óculos de proteção ou viseira, máscara, capote e sapato fechado. Eno caso da sala de reprocessamento dos dialisadores, também chamado de reuso, é necessário utilizar capote impermeável de mangas compridas, botas, luvas de borracha e máscara apropriada contra gases tóxicos.

Torna-se essencial para o bem-estar e a saúde do trabalhador, adotar medidas de proteção e barreira no caso de exposições de pele e mucosas ao sangue ou outros líquidos corporais. Também durante procedimentos invasivos, faz-se necessário o uso de protetores para os olhos, rosto, cabeça e membros $^{12}$.

É fundamental que todos os trabalhadores envolvidos em atividades que tenham algum elemento que represente um tipo de ameaça à sua saúde sejam capacitados a avaliá-los de forma tal que compreendam a importância do uso de medidas de proteção e segurança. A maior responsabilidade sobre o controle dos riscos é do profissional, que os entende e conhece os mecanismos de controle ${ }^{13}$.

Em contrapartida, houve relatos sobre a não-utilização dos EPls, apesar do conhecimento sobre a sua importância, tais como:

0 uso de EPI completo que, às vezes, por ser incômodo, não gostamos ou esquecemos de usar...a gente sempre evita usar algum [...] (T10)

[...] g gente é orientado a usar, mas nem sempre a gente usa da forma correta[...] trabalho acaba exigindo pressa e correria [...] a gente pensa mais no paciente do que em nós próprias, então a gente acaba, muitas vezes, deixando de lado o modo correto de usar devido à necessidade do paciente. (A24)

Tais relatos evidenciam que existe a orientação, porém ainda há limitações quanto à implementação de mudanças tão importantes para a saúde ocupacional. Os relatos permitem inferir que os profissionais de saúde não se julgam suscetíveis aos riscos; muitas vezes, a importância de determinados detalhes somente é entendida em momentos de crise ${ }^{13}$.

Por outro lado, problemas como falta de condições adequadas de trabalho e pressões por produtividade influenciam negativamente, gerando uma desmotivação para a realização das atividades de forma correta ${ }^{13}$.

Entretanto, é importante lembrar que a segurança dos trabalhadores também depende de cada sujeito reconhecer os riscos presentes no ambiente de saúde. 0 trabalhador precisa ter um censo coletivo, desenvolver um sentido de responsabilidade com relação à sua própria segurança e à segurança dos seus colegas e pacientes ${ }^{6}$.
Em alguns momentos foi relatada a dificuldade sobre o uso dos EPIs devido à falta de material disponível no hospital:

$$
\begin{aligned}
& \text { É[...] ter sempre o capote pra gente }[. . .] \text { porque às } \\
& \text { vezes não tem }[\ldots](T 16)
\end{aligned}
$$

No reuso que fica mais complicado...máscara não tem pra todo mundo, a própria[...] que eu acho que é aquela preta[...] nunca vi lá dentro. (T26)

De acordo com a experiência no setor, é fato que ocorre falta de material em alguns momentos, como capotes, máscaras específicas para proteção contra gases tóxicos, etc. Porém, esta é uma realidade brasileira e não pontual deste estudo, 0 que merece reflexão acerca do comportamento destes profissionais, visto que há falha por parte de alguns em relação ao uso dos EPls mesmo quando estes são devidamente fornecidos pelo hospital.

De fato, a aplicação das medidas de proteção não é suficiente para garantir as medidas de prevenção, devendo fazer parte das estratégias as reflexões a respeito das mudanças de comportamento e as causas de acidentes. A não-adesão ou a baixa adesão às recomendações da utilização de barreiras de proteção é uma realidade, o que leva à indagação sobre outros fatores que podem estar contribuindo para este tipo de comportamento ${ }^{14}$.

Em relação às medidas de proteção e segurança relacionados aos riscos ergonômicos, foi notória a citação da reformulação do ambiente de trabalho como principal solução destes riscos, como pode ser visto nas falas a seguir:

[...]melhorar 0 aspecto de pegar pesos, ter uns carrinhos, coisas que melhorem nosso sistema de trabalho. (T7)

Eu acho que o setor por si só deveria ser reformulado, o setor é todo mal montado. (E9)

Aumentar os armários, aumentar o balcão do reuso[...] ]isso aí pra gente é o principal[...] botar um carrinho de manuseio menor para que a gente carregue esses galões[...]porque o box é muito grande...é muita distância entre um box e outro[...] $(A 17)$

0 cenário deste estudo é um setor novo dentro do hospital, porém não se observam nele medidas ergonômicas, como, por exemplo, a altura dos armários e bancadas, além do que os trabalhadores são submetidos a movimentos repetitivos de levantar e abaixar, devido ao posicionamento dos banhos de diálise. 
Por esses motivos, o setor já foi alvo de um estudo ergonômico para possibilitar as mudanças cabíveis e necessárias. Porém, ainda não foram realizadas mudanças organizacionais como medidas para facilitar o processo de trabalho.

Segundo a legislação, os agentes ergonômicos causam riscos à saúde do trabalhador e costumam estar presentes nos ambientes de trabalho $0^{5}$. Cabe ressaltar a importância de se atentar para os procedimentos que são executados de forma repetitiva e monótona e em ritmo acelerado, pois são geradores de desgaste do trabalhador, expondo-o a riscos de acidentes, 0 que sugere a reorganização das atividades ${ }^{14}$.

Além disso, o ambiente interno da instituição deve ser questionado, se os mesmos são saudáveis ou não e se as condições de trabalho são as mais desejáveis para que os trabalhadores possam exercer suas atividades sem risco ou com risco reduzido ${ }^{6}$.

Ainda no contexto desta categoria, houve relatos sobre a educação em saúde aos trabalhadores de enfermagem como medidas de proteção e segurança, conforme os relatos a seguir:

[...] treinamento e esclarecimento também ao próprio funcionário, como se abaixar, evitar aquele posicionamento[...]pelo menos treinar uma forma de eles atenderem os pacientes com um pouco mais de segurança. (E9)

[...]palestras[...] eventualmente sobre os riscos, para a gente estar sempre atento. (A14)

No hospital em estudo, verifica-se a presença de educação em saúde em seus diversos setores. Porém, por tratar-se de um hospital universitário de grande porte, nem sempre é viável a discussão sobre assuntos específicos de cada setor, como a $H D$, e sim sobre assuntos gerais, pertinentes à maioria dos trabalhadores do hospital.

Neste contexto, é importante ressaltar que a educação não deve ser vinculada somente à passagem de conhecimento, mas sim à possibilidade de ampliação da participação política dos trabalhadores ${ }^{6}$.

\section{A influência dos riscos ocupacionais na saúde dos trabalhadores de enfermagem}

A relação entre as exposições ocupacionais e o aparecimento de doenças já é conhecida desde a antiguidade. Logicamente que os acidentes de trabalho e as doenças ocupacionais podem ser mais ou menos frequentes de acordo com a utilização ou não das medidas de proteção e segurança, além do tipo de trabalho exercido pelos trabalhadores.

Nesta categoria foi abordado de que forma os riscos ocupacionais poderiam influenciar a saúde dos trabalhadores da equipe de enfermagem. Encontraram-se relatos sobre a possibilidade de contaminação por agentes biológicos, tais como os vírus da hepatite $\mathrm{B}$ e C, e também pelo HIV, como podemos analisar nas falas a seguir:

$$
\begin{aligned}
& \text { Pode causar }[. . .] \text { pode terminar com um } \\
& \text { HIV }[. . .](A 23)
\end{aligned}
$$

Você pode adquirir uma doença contagiosa que vai te causar problema para o resto da vida[...] como a hepatite, o próprio $H I V[. .].(E 1)$

$$
\begin{aligned}
& \text { Ah, posso me contaminar com hepatite, } \\
& H I V[\ldots](T 18)
\end{aligned}
$$

Em estudo realizado ${ }^{15}$, constatou-se que a HD é o setor com maior frequência de acidentes, cujos procedimentos são constantes, com alto percentual de hepatite $B$ e $C$ entre os dialisados. Dentre os fluidos corporais, tem-se reconhecido 0 sangue como o mais importante veículo de transmissão ocupacional dos vírus da hepatite $\mathrm{B}$ e $\mathrm{C}$ e do $\mathrm{HIV}^{16}$.

Segundo estudos ${ }^{2}$ prospectivos com trabalhadores da saúde, estima-se que o risco médio para transmissão do HIV, após exposição percutânea, é aproximadamente de 0,3\%, e, após exposição de mucosas, de 0,09\%.

A consequência da exposição ocupacional aos patógenos transmitidos pelo sangue não está somente relacionada à infecção. A cada ano, milhares de trabalhadores da saúde são afetados por trauma psicológico que perduram durante os meses de espera dos resultados dos exames sorológicos. Dentre outras consequências, estão ainda as alterações das práticas sexuais, os efeitos colaterais das drogas profiláticas e a perda do emprego ${ }^{16}$

Os sujeitos do estudo também relataram os problemas musculares, especialmente os relacionados com a coluna, como consequência à exposição aos riscos ergonômicos, como se observa a seguir:

E outra coisa que influencia na saúde é esse lance da postura [...] se você acaba tendo uma lesão na coluna....com certeza vai influenciar na sua saúde fora do ambiente de trabalho[...](T11)

[...] eu já vejo que eu já estou com uma idade, já passei dos 50[...] meio século[...] e sinto sim a minha coluna. Eu vejo que esse setor de trabalho é um pouco puxado pra mim [...](T13)

Pode causar um problema na coluna, uma lesão, de repente, irreversivel. Então acho que vai interferir na sua vida como um todo [...](E1) 
Como o setor não oferece um ambiente ergonômico, e também em virtude da própria organização das atividades, é rotineiro o fato de que alguns trabalhadores, principalmente técnicos e auxiliares de enfermagem, apresentem lesões ao nível de coluna vertebral e fiquem restritos às realizações de atividades que não sejam ainda mais prejudiciais à sua saúde. Além disso, tantos outros trabalhadores dessas categorias estão de licença médica, afastados de suas atividades laborais devido aos mesmos problemas.

As afecções do aparelho locomotor constituem importante causa de morbidade e de incapacidade de adultos, e sua importância médico-social e econômica têm chamado a atenção de planejadores e administradores de saúde e de seguridade social no mundo inteiro ${ }^{17}$.

Os problemas respiratórios, oriundos dos riscos químicos que, neste caso, correspondem à exposição ocupacional a agentes esterilizantes, foram bastante mencionados pelos entrevistados, conforme as falas a seguir:

[...]pode terminar com alguma doença respiratória também [...]porque já teve funcionário que já saiu do setor por causa de doença respiratória [...]por causa do material que a gente manipula e manipula por muito tempo[...] (A23)

Sobre o produto, ele irrita a garganta, a nossa saúde, ele dá tosse na gente [...] ele pode mexer no meu pulmão prematuramente e amanhã estar com o pulmão bem atacado[...](T3)

Entre os principais agentes químicos que causam patologias de origem ocupacional, na área hospitalar, estão as drogas antineoplásicas, os agentes esterilizantes e os gases anestésicos, que podem causar desde dermatites e problemas reprodutivos até neoplasias ${ }^{12}$.

A exposição a estes agentes é potencialmente prejudicial aos processos reprodutivos, e deve-se minimizar essa exposição principalmente em trabalhadoras grávidas ou que planejam engravidar ${ }^{12}$. Isso é relativamente importante visto que o maior quantitativo de trabalhadores da equipe de enfermagem do setor é do sexo feminino.

Além do exposto acima, o hipoclorito de sódio pode ocasionar queimaduras oculares que provocam sérios danos à superfície ocular e, muitas vezes, resultam em redução permanente da visão ${ }^{12}$.

\section{CONCLUSÃO}

Este estudo possibilitou caracterizar a percepção dos profissionais de enfermagem quanto aos riscos ocupacionais em uma unidade de tratamento dialítico, conforme especificado anteriormente nos objetivos.
Da coleta de dados foram extraídas três categorias que demonstraram desde o conhecimento dos trabalhadores sobre os riscos ocupacionais até a descrição das consequências destes riscos para a sua saúde.

A partir da análise dos dados, evidenciamos que os trabalhadores do setor estudado conhecem a maior parte dos riscos ocupacionais ali existentes, visto que apenas o risco físico não foi citado nas entrevistas. Em relação às medidas de proteção e segurança, observamos que os trabalhadores se detêm, na maior parte das vezes, àquelas relacionadas aos riscos biológicos e ergonômicos, em detrimento dos demais.

Porém, apesar do conhecimento dos riscos e das medidas de proteção e segurança, não há aplicação prática destas medidas com vista à diminuição da exposição aos riscos e até mesmo das doenças ocupacionais. Este fato é bastante preocupante e digno de uma investigação mais aprofundada objetivando a revisão de estratégias de sensibilização do profissional de saúde perante a prevenção de riscos e doenças ocupacionais.

Acreditamos que seja necessário investir no processo educativo, o que também foi citado pelos próprios sujeitos deste estudo, e em prevenção e controle do ambiente de trabalho, através de treinamentos, cursos e palestras, visando reduzir a exposição aos riscos e prevenir o surgimento das doenças ocupacionais.

Cabe ainda, corroborando alguns autores ${ }^{18}$, referir a importância do Serviço de Saúde do Trabalhador para acompanhar as condições de saúde e as do ambiente de trabalho, implementando medidas de promoção e prevenção da saúde dos trabalhadores.

Esperamos, na particularidade deste estudo, que seja iniciada uma nova era do conhecimento, numa visão integrada, em que o conhecimento dos riscos e a aplicação das medidas de proteção e segurança sejam ferramentas de melhoria da qualidade de trabalho e de vida dos trabalhadores da equipe de enfermagem.

\section{REFERÊNCIAS}

1. Mendes R, Dias EC. Da medicina do trabalho à saúde do trabalhador. Rev Saude Publica 1991;25: 341-49.

2. Mendes R. Patologia do trabalho. Rio de Janeiro (RJ): Atheneu; 1995.

3. Saúde e trabalho: desafio para uma política. Rio de Janeiro (RJ): ABRASCO; 1991.

4. Ministério do Trabalho e Emprego (BR). Portaria 3214, 08 de junho de 1978. Aprova as Normas Regulamentadoras - NR do Capítulo V, Título II, da Consolidação das Leis do Trabalho, relativas a Segurança do Trabalho. NR n 9- Riscos ambientais. Disponível em: http:// unesp.br/pgsst

5. Bulhões I. Riscos do trabalho de enfermagem. Rio de Janeiro(RJ): Folha Carioca; 1994. 
Riscos ocupacionais na perspectiva da enfermagem Silva MKD, Zeitoune RCG

6. Alan MM, César-Vaz MR, Almeida T. Educação ambiental e o conhecimento do trabalhador em saúde sobre situações de risco. Cienc Saude Colet 2005; 10 (sup): 39-47.

7. Balsamo AC, Barrientos DS, Rossi JCB. Estudo retrospectivo dos acidentes de trabalho com exposição a líquidos corporais humanos ocorridos nos funcionários de um hospital universitário. Rev Med HU-USP 2000; 10 (1): 39-45.

8. Marziale MHP, Nishimura KYN, Ferreira MM. Riscos de contaminação ocasionados por acidentes de trabalho com material pérfuro-cortante entre trabalhadores de enfermagem. Rev Latino-am Enfermagem. 2004 jan/fev; 12 (1): 36-42.

9. Xelegati R, Robazzi MLCC. Riscos químicos a que estão submetidos os trabalhadores de enfermagem: uma revisão de literatura. Rev Latino-am Enfermagem, 2003 maio/jun; 11(3): 350-56.

10. Augusto LGS, Freitas CM. 0 princípio da precaução no uso de indicadores de riscos químicos ambientais em saúde do trabalhador. Cienc Saude Colet 1998; 3 (2): 85-95.

11. Oliveira BRG, Murofuse NT. Acidentes de trabalho e doença ocupacional: estudo sobre o conhecimento do trabalhador hospitalar dos riscos à saúde de seu trabalho. Rev Latino-am Enfermagem 2001 jan; 9(1): 109-15.
12. Almeida CB, Pagliuca LMF, Leite ALAS. Acidentes de trabalho envolvendo os olhos: avaliação de riscos ocupacionais com trabalhadores de enfermagem. Rev Latino-am Enfermagem 2005 set-out; 13(5): 708-16.

13. Agência Nacional de Vigilância Sanitária - ANVISA. Biossegurança. Rev Saude Publica 2005; 39(6): 989-91.

14. Balsamo AC, Felli VEA. Estudo sobre acidentes de trabalho com exposição aos líquidos corporais humanos em trabalhadores da saúde de um hospital universitário. Rev Latino-am Enfermagem 2006 maio/ jun; 14(3): 346-53.

15. Ciorlia LAS, Zanetta DMT. Hepatite C em profissionais da saúde: prevalência e associação com fatores de risco. Rev Saude Publica 2007; 41 (2): 229-35.

16. Canini SRMS, Gir E, Hayashida M, Machado AA. Acidentes pérfurocortantes entre trabalhadores de enfermagem de um hospital universitário do interior paulista. Rev Latino-am Enfermagem 2002 mar-abr; 10 (2): 172-78.

17. Mendes R. 0 impacto dos efeitos da ocupação sobre a saúde de trabalhadores. Rev Rev Saude Publica 1988 ago; 22 (4).

18. Farias SNP, Zeitoune RCG. A qualidade de vida no trabalho de enfermagem. Escola Anna Nery Rev Enferm 2007 set; 11(3): 492. 